\title{
Dynamics of vector rogue waves in a fiber laser with a ring cavity: supplementary material
}

\author{
S.A. Kolpakov, ${ }^{*}$ H. KBashi, and S. V. SergeyeV \\ School of Engineering and Applied Science, Aston University, Aston Triangle, Birmingham, B4 7ET, UK \\ *Corresponding author: s.kolpakov@aston.ac.uk \\ Published 1 August 2016
}

This document provides supplementary information to "Dynamics of vector rogue waves in a fiber laser with a ring cavity," http://dx.doi.org/10.1364/optica.3.000870. The material is organized as follows: in the first section, we provided a short theoretical background with the reference to the previously published system of equations. The second section contains the experimental setup. In the third one, we have provided useful information about tuning and modification of oscillating regimes in the laser and have illustrated some oscilloscope traces for the regimes discussed in the article. Finally, the last two sections contain the algorithms processing as well as the figures and annotations which from our point of view may be useful for the consideration of the procedures used for the data processing as well as for a further discussion of the presented in the article results. (C) 2016 Optical Society of America

http://dx.doi.org/10.1364/optica.3.000870.s001

1. The theoretical background of the oscillator. As it follows from the distributed vector model [1] for the ring laser without saturable absorber and fundamental frequency of oscillation $\boldsymbol{F}$ the system of equitation has different eigenfrequencies, some of them $\left(\Omega_{3} \sim 0.01 F\right.$ and $\left.\Omega_{4} \sim 0.001 F\right)$ depend on pump power and another $\left(\Omega_{1} \sim 0.1 F\right)$ on the ratio between the birefringence induced in the cavity and the ellipticity of the pump.

In a long oscillator ( $\sim 600$ meters) the eigenfrequency $\Omega_{4}$ $(\sim 1 / 1000$ of the fundamental frequency) does not excite (see Fig. 1 ) mostly because of a low Q-factor which means the RF peak is just wider than the difference between the fundamental frequency and the $\Omega$.

The resonance interaction between the excited eigenfrequencies and the fundamental frequency leads to new regimes of oscillation which results in different regimes of pulsations. The switch between these regimes was realized by changing the ellipticity of the pump and the birefringence in the cavity.

2.1 The procedure in general. After both polarization controllers have been set in appropriate positions, we have recorded oscilloscope traces with the resolution of $0.1 \mathrm{~ns}$ per point and the length of 50 Mega samples each one. The speed of the pulses has been estimated from the tilt of the traces (see section four). To detect RW patterns a digital filter has been applied to the matrix. The angles of the polarization controllers were measured relatively to the vertical position of a controller knob. The positions of both polarization controllers were changed in the range between - 90 and 90 degrees with the step of 10 degrees. The tilt of the traces was the origin of aliasing obstructing the analysis of the patterns. Finally, to remove the aliasing, the images have been rotated to change the laboratory system of reference to the system of reference related to the RW event. To detect RW patterns a digital filter has been applied to the matrix (see for example right insets (labeled with " $b$ ") in the first rows in Figs. 4-8 in the main text).

2.2 The experimental setup. A $1480 \mathrm{~nm}$ laser diode FOL14xx series with a built-in isolator has been used to pump the cavity via a 1480/1550 wavelength division multiplexer (WDM). The supplied pump power was measured after the pump polarization controller (PPoC), and the WDM just before the active fiber doped with $\mathrm{Er}^{3+}$ (Liekki Er80-8/125). The laser has a threshold of $16 \mathrm{~mW}$; the supplied pump power was $18.4 \mathrm{~mW}$, remaining the same during all the experiment. This value was slightly higher than the lasing threshold (see left inset in Fig. 1). The signal has been detected using an InGaAsUDP-15-IR-2_FC detector with a bandwidth of $17 \mathrm{GHz}$ connected to the oscilloscope Tektronix DPO725 which has the sampling rate of $2.5 \mathrm{GHz}$. The oscilloscope has the built-in option of a variable electronic filter for the incoming signal. During the experiments, the bandwidth was set up to be $2 \mathrm{GHz}$. An optical spectrum analyzer Yokogawa AQ6317B was used to record the laser spectra. The resolution of the OSA was $0.02 \mathrm{~nm}$.

The radio frequency (RF) spectrum illustrated in Fig. 1 shows three frequencies. The central peak corresponds to the round trip 
frequency; two satellites labeled as "Polarization" are related to incavity birefringence-mediated instabilities. The frequency of this modulation depended on birefringence induced in the cavity by CPoC. In addition, there are two smaller satellites (see right inset in Fig. 1). The positions of these satellites were not dependent on induced birefringence but they were on a pump power.

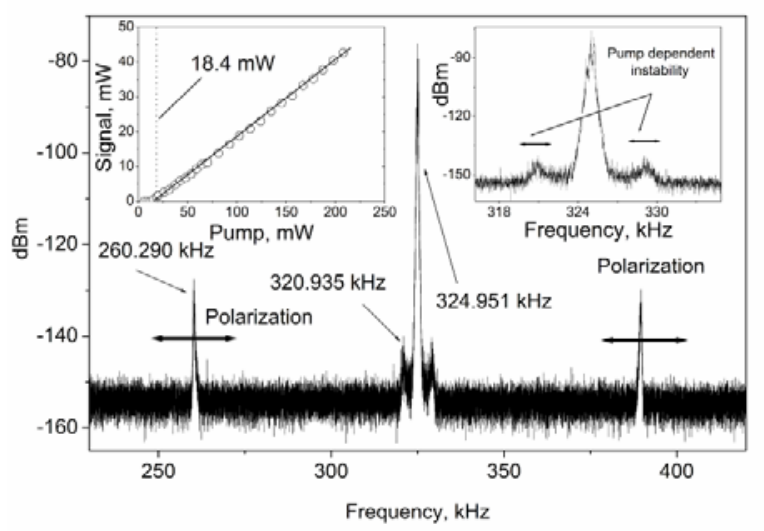

Fig. S1. RF spectrum of the laser. In the left inset the dependence of generated power of the pump power and in right inset the amplified central part of the spectrum.

The changes in RF spectrum when RWs were detected are illustrated in Fig. 2. Note that pump dependent satellites which were in a distance of $\sim 4 \mathrm{KHz}$ (Fig. 1) from the main frequency, have been disappeared. The wide peaks which appeared at the distance of $\sim 1.5 \mathrm{kHz}$ from the central peak in Fig. 2 matched the frequency of "breathing" of the "twins" and "three sisters" patterns. The similar frequency of "breathing" was detected for the "lonely" patterns but in these case the "breathing" of the amplitude was observed for pulses with significant wave height (SWH) rather than for the RWs, due to these patterns have probably a shorter lifetime than the period of the "breathing".

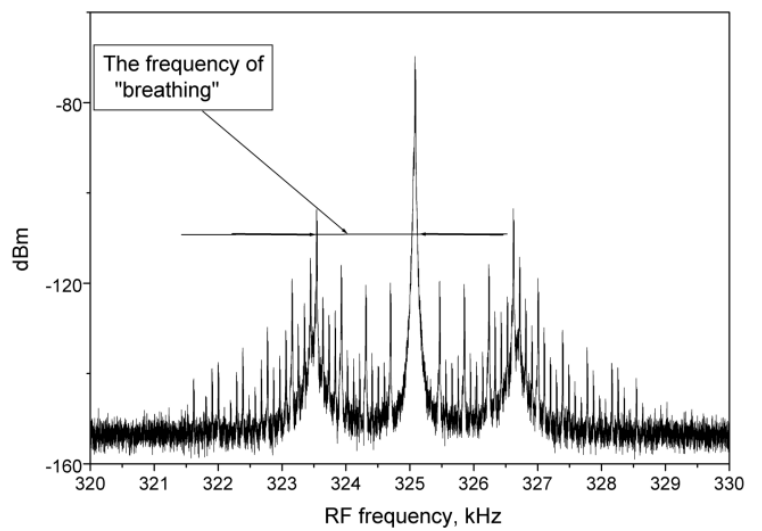

Fig. S2. The RF spectrum when the RW patterns have been identified in the oscilloscope traces. The difference between the main line and the satellite matches the frequency of a "breathing".

\section{The patterns and regimes of lasing.}

The "lonely" pattern. The example of the oscilloscope trace for one of the regimes of lasing where "lonely" FORW patterns have been observed is illustrated in Fig. 3. We have observed that the "lonely" FORW patterns were more common than the "twins" and "three sisters" patterns. The last two have a low likelihood, and appears mostly in the regime similar to soliton rain. The "lonely" pattern also was observed in few occasions along with "cross" and "variable speed" patterns. On all occasions, it was the regime similar to soliton rain (as it shown in Fig. 3).

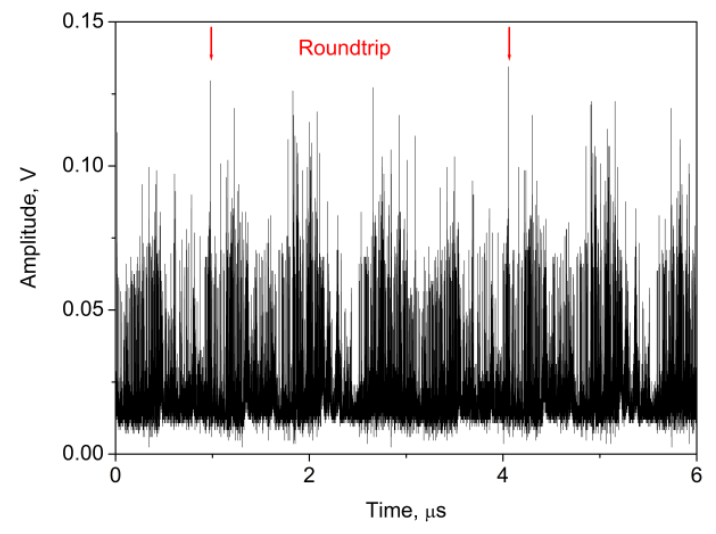

Fig. S3. The oscilloscope traces for the regime with a "lonely" FORW patterns.

The "twins" pattern. The example of the oscilloscope trace for the regime where "twins" pattern have been observed is illustrated in Fig. 4.

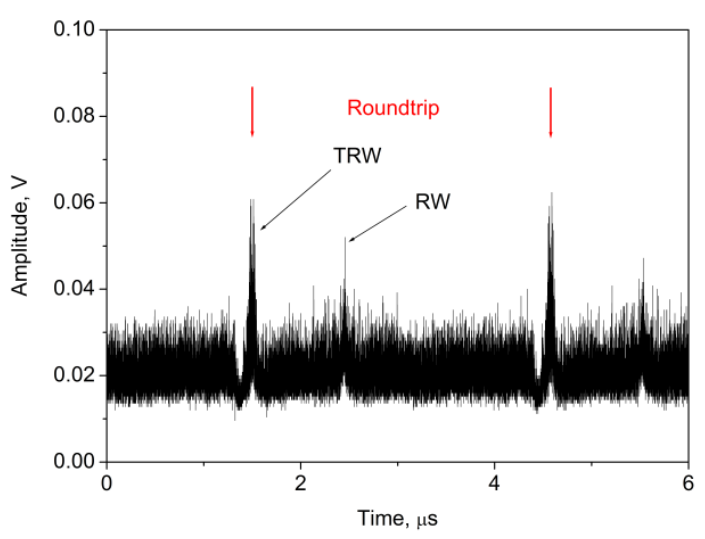

Fig. S4. The oscilloscope trace for the regime where the "twins" pattern was detected. The arrow labeled with "TRW" indicates the place where "twins" pattern has been detected; the arrow labeled with "RW" indicates extremely rare situation when weak and very short-living "lonely" FORW pattern was observed along with the "twins".

The "three sisters" pattern. The oscilloscope trace for the regime when the "three sisters" pattern along with "lonely" patterns have been detected is illustrated in Fig. 5. Unlike in the case of "twins" patterns, these events have been detected between main pulses at multi-pulsing regimes of lasing. These patterns have a relatively short lifetime which was longer than 100 round trips only in few occasions. 


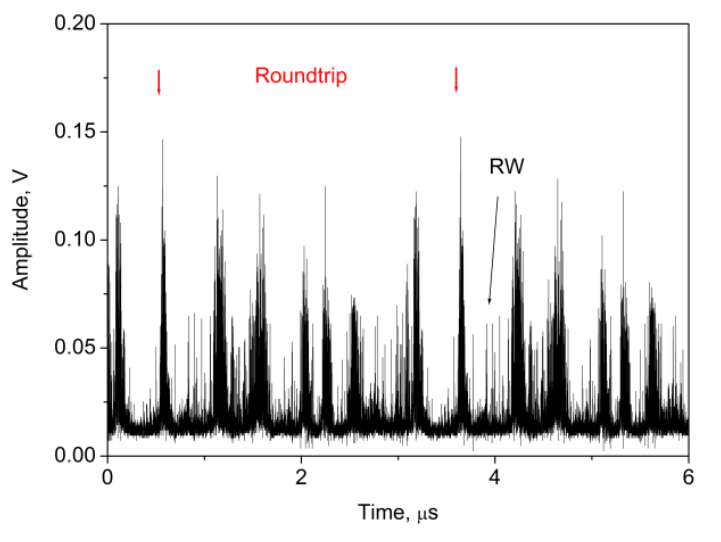

Fig. S5. The oscilloscope trace for the regime when "three sisters" pattern was detected. The arrow with the label "RW" indicates the position of "three sisters" pattern.

4. Data processing, filtering of RW events and removing of aliasing. The speed of light in the fiber depends on the polarization, for this reason, the laboratory system of reference has been changed to the new system of reference where the speed of light for circular polarization was supposed to be zero. The oscilloscope trace was split into segments, the length of each segment was taken equal to one round trip for the wave with the circular polarization ( 30773 pixels of $0.1 \mathrm{~ns}$ each). After that these segments were rewritten as rows of a matrix in the next order: the first roundtrip was placed in a first row, the second in the second one at cetera. In this system of reference, the traces of a pulses propagating with the average speed of light equal to zero (the circular polarization) were represented by vertical lines; meanwhile, the traces of pulses with elliptical or linear polarization were looking tilted.

The examples of traces for two elliptical polarizations are illustrated in Fig. 6. In inset (a) the pulses are moving faster than the average speed of light and the traces of the pulses are tilted clockwise which means the that the pulses were propagating faster than the average speed of light and the light was polarized mostly along the fast axis; in the right inset (b) the traces of the pulses are tilted counterclockwise which means that the light was polarized mostly along the slow axis and the pulses were propagating slower than the average speed of light.

The matrix of a filter has the same dimensions as the matrix of data. Initially, zero value (white color) was assigned to all the elements of this matrix. When the amplitude of the pulse was higher than eight standard deviations from the mean value of the distribution function, the value of " -1 " was assigned to a corresponding matrix element (black color), otherwise, the value remained zero and the pixel white.

In Fig. $6 \mathrm{~b}$ the black and white digital filter was combined with the image matrix (only for illustration). The image of the filter matrix (Fig. 6b) has black points in places where the amplitude has surpassed the RW threshold; the black arrows in Fig. 6b are indicating the RW events.

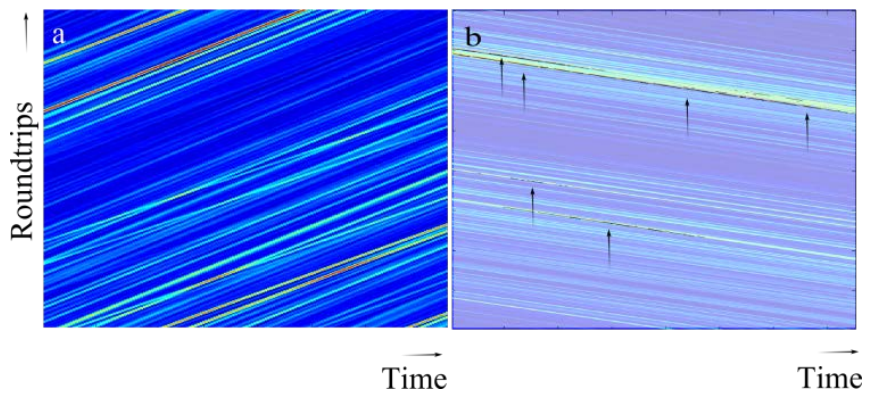

Fig. S6. a-The example of pulses propagating faster than the average speed of light and $b$ - the pulses propagating slower than the average speed of light. No RWs have been detected in the left pattern, meanwhile in the right one, the applied digital filter has indicated the presence of FORW (the black arrows indicate where we have spotted FORW patterns).

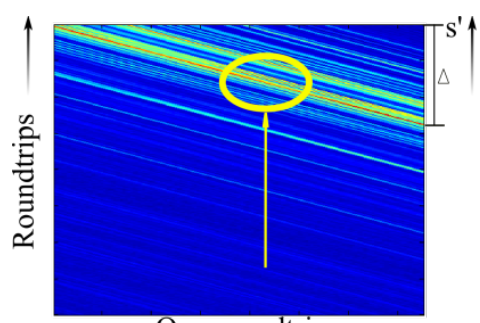

One roundtrip

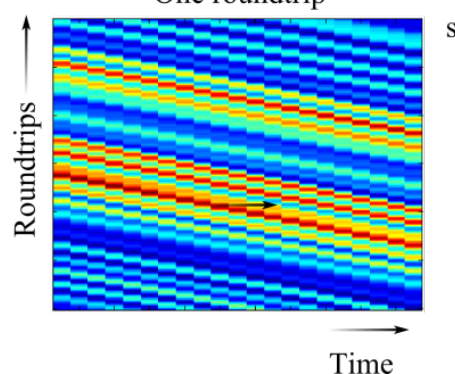

Time

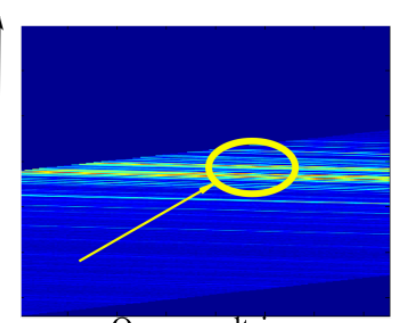

One roundtrip

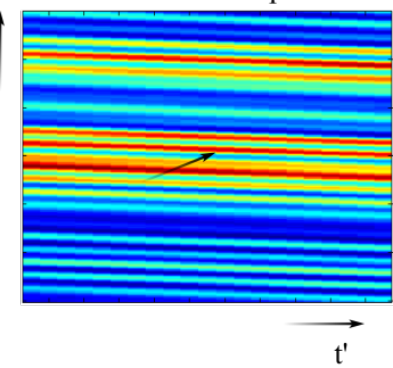

Fig. S7. The correction of a tilt. The left column original data the right column the data in a system of reference ( $\left.s^{\prime}, t^{\prime}\right)$; the matrix, in this case, has twice of rows than the original one. The second row shows the amplified encircled area, the aliasing is clearly seen in the left low inset. Black arrows indicate the direction of propagation.

Obtained in this way, images have aliasing because of a finite time of the oscilloscope sampling. For removing the aliasing the images have been tilted (compare left and right insets in Fig. 7). The tilt was made using the Lorentzian transformation of the system of coordinates to change it to the system of coordinates of the pulse $\left(s^{\prime}, t^{\prime}\right)$. Although due to the discrete time of sampling the traces of the pulses still looks slightly tilted. Insets in a second row of the image show the amplified encircled areas in the figures in the top row.

5. Estimation of propagation speed for a solitary RW pattern. The speed of light, as well as the polarization of it, have been estimated using the temporal width of the pixel ( $0.1 \mathrm{~ns})$ and the length of the whole cavity was $\sim 615$ meters. The interval $\Delta$ (see left inset in the first row in Fig. 7) represents how many roundtrips (length of each was $3.0773 \mu \mathrm{s}$ ) the pulse has been circulated to acquire a delay of one round trip ( $\sim 615$ meters). 

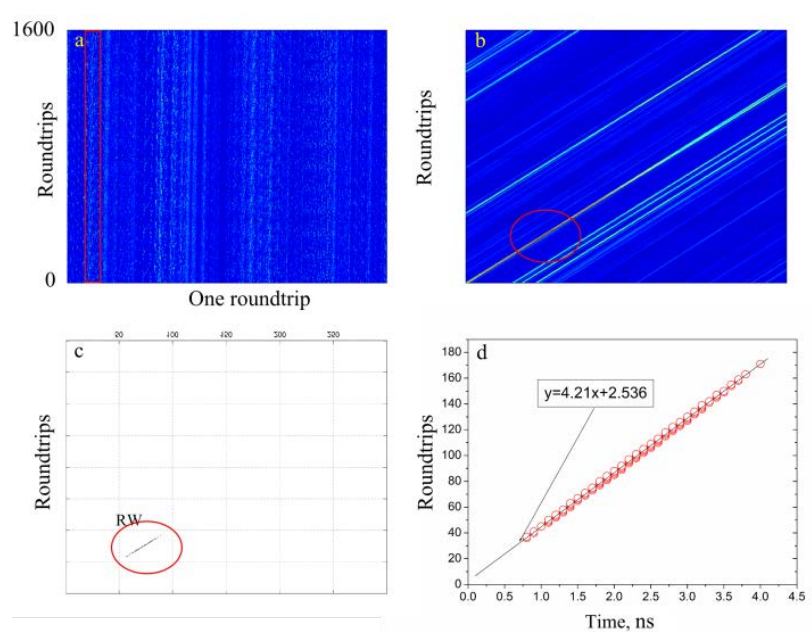

Fig. S8. The procedure of estimation of the relative speed of the pulse: a-whole pattern; b-the amplified area inside of the red square, c-the results of the digital filtering and d-the linear fitting for the dataset illustrated in "c".

The procedure of estimation of the speed is illustrated in Fig. 8 using the same example of the "lonely" pattern which was illustrated in the main text. After using the digital filter, the FORWs were detected in the highlighted with the red rectangle area (see Fig. 8a) and the highlighted area has been amplified (see Fig. 8b). The result of the digital filtering for the amplified area is shown in Fig. 8c; the encircled area, shows the place where the "lonely" FORW pattern was detected (compare insets b and $c$ in Fig. 8). After amplifying the area of interest, the points with the value of "1 " were subtracted from the matrix of a filter to an array and fitted with a linear polynomial function. The coefficient before "x" (we have used "x" and " $y$ " instead of "t" and "z" due to the fitting was made in pixels and roundtrips) indicates that every 4.21 round trips $(\sim 13 \mu \mathrm{s})$ the pulse has advanced of $0.1 \mathrm{~ns}(\sim 2 \mathrm{~cm})$ in reference to a pulse with the average speed of light, this gave us an estimation of the relative speed of the pulse in the pattern to be $\sim 1538 \mathrm{~m} / \mathrm{s}$.

\section{References}

1. S. A. Kolpakov, S. V. Sergeyev, Yu. Loiko, N. Tarasov, V. Kalashnikov, and G. P. Agrawal, "Resonance vector mode locking," http://arxiv.org/abs/1508.05933 